\title{
Effective integration of computational tools into Chemical Engineering studies at an international level
}

\author{
Claudia Roman', Miguel Ángel Delgado, Francisco Lemos², Maria Amélia Lemos², \\ Jorge Ramírez ${ }^{3}$, Angela Danila ${ }^{4}$, Moisés García-Morales ${ }^{1 *}$
}

${ }^{1}$ Department of Chemical Engineering, Physico-chemistry and Materials Science, Universidad de Huelva, Spain, ${ }^{2}$ Department of Chemical Engineering, Instituto Superior Técnico de Lisboa, Portugal, ${ }^{3}$ Department of Chemical Engineering, Universidad Politécnica de Madrid, Spain, ${ }^{4}$ Department of Chemical Engineering in Textile and Leather, Gheorghe Asachi Technical University of Iași, Romania.

\begin{abstract}
Current Higher Education students have grown up in a society characterized by the massive use of information technologies, which affects the way they expect to acquire new knowledge. In Chemical Engineering studies, in particular, traditional problem solving methods tend to bore students and, as a result, do not yield efficient learning. Fortunately, there exists a large list of software packages with specific Engineering application which, if properly used, may help create a better learning environment.

Under the above premise, a project is being conducted, between 4 Higher Education institutions from 3 different countries (Spain, Portugal and Romania), on the effect that the integration of computational tools may exert on the students' knowledge acquisition and predisposition to learn. We also aim to establish a comparative evaluation of the advantages and drawbacks of different computer software when facing typical Chemical Engineering problems. From our survey results and students' comments we conclude that, in general, the new methodological approach engaged their interest more than the traditional one, and helped them gain knowledge on the working principles of simulations. Moreover, the use of computer software in the classroom is acknowledged by the great majority of the students as a key skill which may improve their employability prospects.
\end{abstract}

Keywords: Chemical Engineering; educational computer software; ICT competences; internationalization. 


\section{Introduction}

It is well known that our society is ruled by an increasing and generalized use of information technologies at all levels, a situation which has also reached the Higher Education level. In fact, most students expect to use electronic devices as the only way to access information when learning. Traditional masterclasses tend to bore them. Thus, a successful integration of technologies into their courses seems a very good approach to engage them. Moreover, in the Chemical Engineering discipline, the use of computers in process control, simulation and design has become an essential technical competence that must be acquired by all future professionals (Gillett, 2001).

The matters which constitute the core of Chemical Engineering studies, such as Unit Operatations, Chemical Reactors, Fluid Mechanics, Process Control and Optimization, etc., involve a great load of complex and tedious calculations. Traditional problem-solving by hand makes the students feel uncomfortable and does not always facilitate a deep understanding. Fortunately, the integration of commercial software in the classroom may help design more appealing teaching programs. Use of software enables more realistic open-end problems, which yield a better comprehension of the fundamentals, and allows the students to investigate the effect of the process variables with more flexibility. Table 1 gathers some information on the software packages used in the present experience. The selection is based on two issues: a) as one of the project's target is to compare strengths and weaknesses, it was necessary to provide a list of software with different characteristics in terms of graphical interface, difficulty, relative widespread use, programming, etc.; b) availability and expertise of the teaching staff participating in this project.

Table 1. List of software package used in this project.

\begin{tabular}{llllll}
\hline Software & Company & Availability & Type & Programming & Difficulty \\
\hline MS Excel & Microsoft & High & Spreadsheet & Optional & Low/medium \\
Python & $\begin{array}{l}\text { Python Soft. } \\
\text { Foundation }\end{array}$ & Free & $\begin{array}{l}\text { Programming } \\
\text { language }\end{array}$ & Yes & High \\
Mathcad & PTC & Low & Engineering maths & Optional & Medium/high \\
Aspen Plus & Aspen Tech & Medium & Process simulator & No & Medium \\
\hline
\end{tabular}

The use of individual software packages in Chemical Engineering teaching has been reported (Binous, 2008; Parulekar, 2006; Roman and García-Morales, 2019; Wankat, 2006). However, to the best of our knowledge, there is still a lack of studies which compare their adequacy for specific matters. A comparative evaluation on the use of different 
computer software carried out on core matters of Chemical Engineering by a coordinated team of academics would allow discovering the specific features that make each of them more suitable than the others for a certain type of problem in this field.

Under the above hypothesis, a project on the integration of computational tools into core matters of Chemical Engineering studies is being developed between 4 Higher Education institutions from 3 different countries (Spain, Portugal and Romania), aimed to:

- Establish a comparative analysis of the strengths and weaknesses of each software package.

- Find out whether the approach enabled a more efficient learning.

- Assess the students' predisposition to learn with the application of the method.

\section{Methodological approach}

One of the most positive aspects of this project is the constitution of a work team composed of 7 academics from Spain, Portugal and Romania, all of them working in the field of Chemical Engineering. Due to different affiliations, the team was organized into 5 groups. In any case, the methodology herein described corresponds to the joint project and not to the individual activity carried out at every institution.

\subsection{Preparation of proposals and surveys}

In September 2019, each group was required to propose 2 activities, involving the effective integration of computer software for improving the students' comprehension and strengthening ICT competences. A preliminary videoconference enabled the participants to agree on both topics and software packages.

The project coordinator prepared two different surveys: a) the first one was aimed to know about the students' satisfaction; b) the second survey was intended to gather information on the teachers' background. Both students and teachers were asked to rate every item between 1 (completely disagree) and 5 (completely agree). At the end of the documents, a blank for additional comments was provided. Finally, an editable MS Word template was also provided for the participants to report the results.

\subsection{Activities implementation and evaluation}

Each lecturer was free to decide whether the activities should be conducted individually or in groups; in the classroom, computer lab or at home; with one software or several, etc. In most of cases, a previous software training was necessary because the students only had basic knowledge of them. Both transversal and conceptual competences should be assessed through the corresponding assignments, which should be marked between 0 (lowest grade) and 10 (highest grade). 


\subsection{Project framework and analysis of the results}

In brief, the following questions have guided the process of evaluating the software implications on the learning process: a) did it give the chance for an easy graphical solution?; b) did it provide any professional skills?; c) did it allow for natural notation in terms of writing equations?; d) did it enable accuracy and time-saving as compared to the traditional hand problem-solving method?; e) did the creation of code/worksheet entail a big concern to the students?; f) did it allow for easy editing of existing code/worksheet so as to vary the variable of study?; g) was the analysis of both experimental data and theoretical models possible?

Moreover, in relation with the two other goals, surveys and assignments grades have also contributed to collect information on students' satisfaction and performance.

\subsection{Final report}

At the end of the project, the coordinator will have to submit a final report to the funding organization (Vicerrectorado de Innovación y Empleabilidad, UHU) so they can evaluate whether the project has yielded effective learning and has fulfilled the stated objectives.

\section{Results and Discussion}

\subsection{On the teaching staff}

From the lecturers' survey (7 lectures), we highlight the following information:

- In general, all the participants have previous experience on the integration of computational tools into Chemical Engineering courses, and they have learnt how to use use all the software by themselves.

- However, some of them have never participated on a teaching project on this topic, and the majority of the team has never published results on this type of methodological approaches.

- Most of them admit that using software has implied more effort than traditional problem solving.

- Fortunately, there seems to be a common perception that the project has been well received by the students and has enhanced their interest and participation.

- In general, their home institutions provide the computational facilities needed, although sometimes they make use of the students' own laptops.

\subsection{Adequacy of the software used in specific applications}

A Python code based on elastic collisions between gas molecules was developed for one of the activities. It enabled the students to run a set of simulations on the chemical kinetics 
laws from which they were asked to analyze the results in the context of the kinetics theory of gases. Figure 1a illustrates the simulation of a first order reaction. As a drawback, programming the code was not an easy task. So, the instructor had to develop the code and then make it available to the students. Fortunately, the code can be transformed into executables for the students to download and use at home. In this way, Python allowed the creation of a tailored application, commercially unavailable, for the simulation of a specific process with educational purpose. Also, it is possible to edit the code so that the application is run in "game" mode, which would increase further the students' motivation. Other positive aspects of Python are that it is open source and there is a large number of specialized libraries available. For the students to acquire a more solid knowledge, the lecturer who wrote the code recommends to use Python in a coordinated way with a more traditional approach.

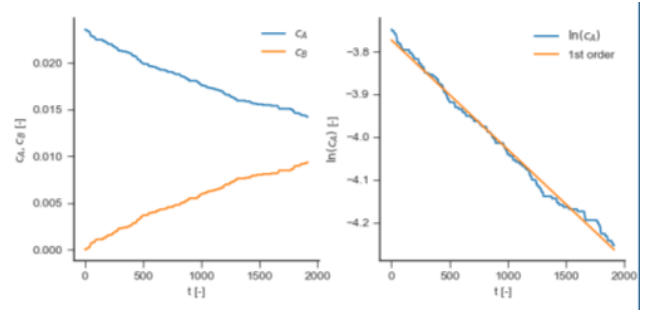

(a)

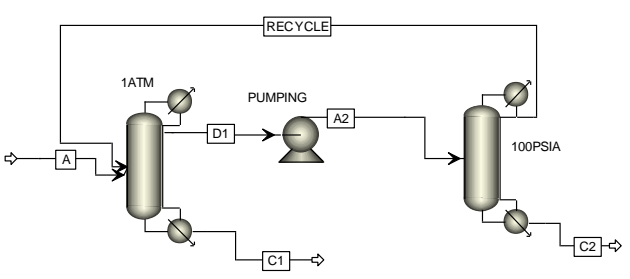

(b)

Figure 1. Selected examples of the digital teaching material developed for the project.

Another activity, involving the separation of azeotropic mixtures by fractional distillation, was carried out using the process simulator Aspen Plus. One example, for a separation of water and methy-ethyl-ketone by the pressure-swing method, is shown in Figure 1b. Aspen Plus provided great flexibility for implementing a "sensitivity" analysis aimed at an easy and fast manipulation of the key process variables, and for exploring "what-if" scenarios, with reduced learning time. Another positive feature is the availability of the latest thermodynamical models and data libraries, which provide accurate designs. However, given that the students do not perceive the details of the calculations, there exists a big concern about the so-called "black-box" effect, that is, a superficial understanding of the problem. To minimize that, the Mathcad software will be used in parallel with Aspen Plus simulations in a further activity next semester. It will allow us to create a more efficient learning environment in the computer lab. The main disadvantage about Mathcad is its typically low availability in Higher Education institutions.

MS Excel was used to implement three other activities. It proved to be successful in applications which involved repetitive calculations and which required to organize a big amount of data, obtained from lab experiments, in a simple way. In our project, for example, MS Excel was used to determine the surface tension coefficient of three 
surfactants at three different concentrations, by the gas bubble method. However, MS Excel is a very versatile tool which enables more advanced applications if used conveniently. This is exemplified by one of our approaches consisting in the design of a stationary single or multi-effect evaporator through the implementation of a solving algorithm involving the powerful logic functions. The tool provided flexibility for the study of the main process variables and allowed fast editing, thus enabling easy understanding of the evaporator performance. Finally, evidences of the above reported flexibility of this tool are manifested by another proposal consisting in a transient process taking place in a double tube heat exchanger, for which the time dependent temperature profiles were examined. Some other advantages of MS Excel are: it is always available at the University computer labs; almost all the students have some previous knowledge on this software, what facilitates a lot the activities implementation; everyone has MS Excel installed in his/her own laptop. However, if compared to previous software packages above described, its graphical interface is less user-friendly, lacks of natural notation and it has more limitations to develop complex calculations. A summary with the 5 activities so far completed is shown in Table 2.

Table 2. Summary of activities developed during the Winter semester 19-20.

\begin{tabular}{|c|c|c|c|c|}
\hline Activity title & Matter & Year & Software & $\mathbf{n}^{*}$ \\
\hline $\begin{array}{l}\text { Unsteady-state operation of a concentric tube heat } \\
\text { exchanger }\end{array}$ & $\begin{array}{l}\text { Heat } \\
\text { transfer }\end{array}$ & $3 \mathrm{rd}$ & MS Excel & 56 \\
\hline $\begin{array}{l}\text { Improving the understanding of chemical kinetics } \\
\text { using an application created with Python }\end{array}$ & $\begin{array}{l}\text { Chemical } \\
\text { kinetics }\end{array}$ & $1 \mathrm{st}$ & Python & 70 \\
\hline $\begin{array}{l}\text { Complex Distillation Methods for the separation of } \\
\text { azeotropic mixtures }\end{array}$ & $\begin{array}{l}\text { Unit } \\
\text { operations }\end{array}$ & $3 \mathrm{rd}$ & Aspen Plus & 6 \\
\hline Interactive design of an evaporation unit using Excel & $\begin{array}{l}\text { Unit } \\
\text { operations }\end{array}$ & 3 rd & MS Excel & 15 \\
\hline $\begin{array}{l}\text { The influence of some surfactants on the surface } \\
\text { tension coefficient }\end{array}$ & $\begin{array}{l}\text { Applied } \\
\text { chemistry }\end{array}$ & 4 th & MS Excel & 6 \\
\hline
\end{tabular}

\subsection{Students' feedback and main implications on their learning process}

The results of the students' survey (a total of 159 participants, $57.9 \%$ male and $42.1 \%$ female) are summarized in Table 3, in terms of average score \pm standard deviation. All the students belong to Bachelor's Degrees in Chemical Engineering and, as specified in Table 2 , most of them are in the last two years of their study programs. In the overall calculations the same weight was assigned to each activity, no matter the relative number of participants. The surveys evidenced that: 
- In general terms, the use of software catalyzed a better understanding of theoretical contents and made the course more attractive.

- Regardless of the digital tool used and of the home institution where the activity was carried out, the great majority of students considered computer skills as very important for their professional career as well as for their final project.

- Students do not accept as valid the statement "I prefer to solve problems by hand", and they consider that computational methods are easier. In fact, they propose to extend their use to other subjects in their study programs.

- With respect to the previous knowledge of the software, statistics by software type (not shown) showed that Excel got the highest score and Python the lowest.

- The opinions coincide on that the load of digital tools during their studies is only medium.

Table 3. Students' survey results.

\begin{tabular}{ll}
\hline \multicolumn{1}{c}{ Questions } & $\boldsymbol{\mu} \pm \boldsymbol{\sigma}^{*}$ \\
\hline 1. The software used enabled a better understanding of the theoretical contents & $4.05 \pm 0.68$ \\
2. The software used made the subject more attractive & $4.26 \pm 0.67$ \\
3. The software used was familiar to me. I did not need any previous training & $2.93 \pm 0.41$ \\
4. The software used enabled to analyze different alternatives and to decide & $3.99 \pm 0.55$ \\
5. Thich one is best & $4.24 \pm 0.66$ \\
6. I do not like computers, I prefer to solve the problems by hand & $2.20 \pm 0.82$ \\
7. To solve problems by hand is much easier than with software & $2.54 \pm 0.64$ \\
8. To gain computer skills is important with a view to my final project & $4.29 \pm 0.40$ \\
9. Most of my teachers make use of computer software to solve the problems & $2.99 \pm 0.41$ \\
\hline
\end{tabular}

* average \pm standard deviation 
Additionally, the most relevant comments provided by the students are presented:

- "For us to carry out a everyday use of Excel, we'd need more training".

- "I enjoyed the team work. It offered me the chance to get to know my classmates much better".

- "The activity helped leave the usual daily routine".

- "The assignment was ok, but we had some problems handling the software".

- "The graphical interface (Python) was useful to understand the reaction which was taking place".

\section{Concluding remarks}

This is a good example of internationalization of teaching practices at the University level, following one of the priority lines of work of the European Higher Education Area. It is also in line with the promotion of the digital culture.

With regard to the teaching staff, they were qualified in the use of computer software and had previous experience on their integration into Chemical Engineering subjects. Even so, the majority of the team had never published results on these methods. In general, the Engineering lecturers recognize their lack of theoretical formation on the issue as the main concern which makes them feel uncertain and prevent them from taking a step further. They also admitted that preparation of the material took more time and effort than traditional masterclasses.

As for the students, in general they acknowledge whichever digital tool that teachers are willing to use. One of the key phrases was "the activity gave us the chance to leave the usual daily routine...". This summarizes very well the students' feeling, who are annoyed with the traditional learning methods that are most of the times used in the lecture room. Gaining skills which they consider really useful for getting a job (ICT skills) is one of the things they care most. However, the use of digital tools during their studies is less than optimal. Moreover, using computer tools also promoted interpersonal relationships.

\section{Acknowledgements}

M. García-Morales, coordinator of the project "La enseñanza de la Ingeniería Química en el Tercer Milenio: integración efectiva de herramientas computacionales" belonging to XXI Convocatoria de Proyectos de Innovación Docente, acknowledges Vicerrectorado de Innovación y Empleabilidad de la Universidad de Huelva for its financial support. 


\section{References}

Binous, H. (2008). Equilibrium-staged separations using Matlab and Mathematica. Chem Eng Educ., 40(2), 69-73.

Gillett, J. E. (2001). Chemical Engineering Education in the Next Century. Chem. Eng. Tech., 24(6), 561-570.

Parulekar SJ. Numerical problem solving using mathcad in undergraduate reaction engineering. Chem Eng Educ. 2006;40(1):14-23.

Roman, C., García-Morales, M. (2019). Achieving a better understanding of binary azeotropic mixtures distillation through Aspen Plus process simulations. Comput Appl Eng Educ, 27, 1453-1454. Doi: 10.1002/cae.22161.

Wankat, P. C. (2006). Using a commercial simulator to teach sorption separations. Chem Eng Educ., 40(3), 165-172. 